\title{
Quantum noise in optical fibers I: stochastic equations
}

\author{
P. D. Drummond ${ }^{1}$ and J. F. Corney ${ }^{1,2}$ \\ ${ }^{1}$ Department of Physics, The University of Queensland, St. Lucia, QLD 4072, Australia \\ ${ }^{2}$ Department of Mathematical Modelling, Technical University of Denmark, DK-2800 Lyngby, \\ Denmark
}

July 24, 2013

\begin{abstract}
We analyze the quantum dynamics of radiation propagating in a single mode optical fiber with dispersion, nonlinearity, and Raman coupling to thermal phonons. We start from a fundamental Hamiltonian that includes the principal known nonlinear effects and quantum noise sources, including linear gain and loss. Both Markovian and frequency-dependent, non-Markovian reservoirs are treated. This allows quantum Langevin equations to be calculated, which have a classical form except for additional quantum noise terms. In practical calculations, it is more useful to transform to Wigner or $+P$ quasi-probability operator representations. These result in stochastic equations that can be analyzed using perturbation theory or exact numerical techniques. The results have applications to fiber optics communications, networking, and sensor technology.
\end{abstract}

060.4510, 270.5530, 270.3430, 190.4370, 190.5650, 060.2400

\section{Introduction}

The propagation of electromagnetic radiation through optical fibers is the central paradigm of optical communications and sensor technology. It is also a novel physical system, due to the materials processing of fused silica, that leads to single-mode behaviour with extremely low losses. Over short distances (depending on the pulse intensity) the well-known nonlinear Schrödinger equation can describe most optical fibers with great accuracy, and leads to soliton behaviour, as well as to many other effects. Over longer distances, a number of reservoir effects intervene, including attenuation, Raman scattering, and the use of amplifiers and filters to compensate for losses. At the quantum level, both the original nonlinearity and the additional couplings to reservoirs can lead to quantum noise - which modifies the predictions of the classical nonlinear Schrödinger equation.

In this paper, we analyze the effects of quantum noise in fiber optics. This extends and explains in more detail earlier theoretical work in this area, which led to the first prediction 1 国 and measurement ${ }^{3}$ of intrinsic quantum noise effects in optical solitons. The theory given here includes a detailed derivation of the relevant quantum Hamiltonian. We 
include quantum noise effects due to nonlinearities, Raman reservoirs and Brillouin scattering. The Raman/Brillouin noise is modeled using a multiple Lorentzian fit to measured fluorescence data, in order to estimate the Raman gain coefficients. Both gain and loss effects are included. This treatment is unified with theory of gain/loss reservoirs, which was also predicted ${ }^{-}$and observed ${ }^{0}$ to have large effects on soliton propagation. All these reservoirs are treated without using the Markovian approximation, in order to accurately treat the frequency-dependent reservoirs found in practical applications.

The purpose of this work is to lay the foundations of practical methods for calculating and numerically simulating quantum effects in nonlinear optical fibers. These are significant whenever quantum-limited behaviour is important in communications, sensing, or measurement with optical fibers.

We introduce the basic quantum Hamiltonian for an optical fiber in section 2. This gives a Heisenberg equation of motion which reduces to the nonlinear Schrödinger equation in the classical limit. The equation of motion is extended in section 3 to include Raman and Brillouin effects, with gain and absorption processes considered in section 1 . The complete Heisenberg equation in section 5 is the central result of this paper. Stochastic partial differential equations can be derived from the quantum equation, using the phase-space methods outlined in section 6. Applications of these methods to practical examples are reserved for a following paper (QNII)

\section{Nonlinear Schrödinger Model}

The interaction between photons in a fiber is mediated through the dielectric material constituting the fiber. The coupling to the dielectric introduces frequency dependent and time delayed behaviour. The complete Hamiltonian and its derivation have been given in the literature1. 10; we will only go over the salient points here. The starting point is a Lagrangian that generates the classical Maxwell's equations for a one-dimensional dielectric waveguide, and that gives a Hamiltonian corresponding to the dielectric energyt:

$H_{D}=\int d V\left[\frac{1}{2 \mu}|\mathbf{B}|^{2}+\int_{t_{0}}^{t} \mathbf{E}\left(t^{\prime}\right) \cdot \dot{\mathbf{D}}\left(t^{\prime}\right) d t^{\prime}\right]$,

where the electric field $\mathbf{E}=(\mathbf{D}-\mathbf{P}) / \epsilon_{\mathbf{0}}$ includes the polarization response of the dielectric to an incident electric displacement $\mathbf{D}$. The field variables are then quantized by introducing equal-time commutators between the canonical coordinates $\mathbf{D}$ and $\mathbf{B}$. We note that, of course, it is also possible to make other choices of canonical momenta. This choice corresponds to a dipole-coupled $\$ 1$, rather than minimal-coupled fundamental Lagrangian. While different Lagrangians are canonically equivalent, the present choice - originally introduced 22 by Hillery and Mlodinow in applications to dielectric theory - has the advantage of comparative simplicity. The Lagrangian must produce both the correct energy 13 and Maxwell's equations, otherwise the conjugate momenta will contain an arbitrary scaling, leading to incorrect commutation relations 2 造. 


\section{A. Fiber-optic Hamiltonian}

The optical fiber treated will be a single transverse mode fiber with dispersion and nonlinearity. Since boundary effects are usually negligible in experiments, it is useful to first take the infinite volume limit, which effectively replaces a summation over wave-vectors with the corresponding integral. We will start with a single polarization direction (i.e., a polarization preserving fiber). The more general case is summarized elsewhere 14 , and will be treated in detail subsequently. The basic normally ordered, nonlinear Hamiltonian for the fiber in this case is

$\widehat{H}_{F}=\int d k \hbar \omega(k) \widehat{a}^{\dagger}(k) \widehat{a}(k)-\int d^{3} \mathbf{x}\left\{\left[\frac{\Delta \chi^{(1)}(\mathbf{x})}{2 \epsilon\left(\omega_{0}\right)}\right]:|\widehat{\mathbf{D}}|^{2}(\mathbf{x}):+\left[\frac{\chi^{(3)}(\mathbf{x})}{4 \epsilon^{3}\left(\omega_{0}\right)}\right]:|\widehat{\mathbf{D}}|^{4}(\mathbf{x}):\right\}$.

Here $\omega(k)$ is the angular frequency of modes with wave-vector $k$, describing the linear polariton excitations in the fiber, including dispersion. We will assume that $\omega(k)$ describes the average linear response of the fiber, in the limit of a spatially uniform environment. If the fiber is spatially nonuniform, then it is necessary to add additional inhomogeneous terms to the Hamiltonian, of generic form $\Delta \chi^{(1)}(\mathbf{x})$. As usual, $\epsilon\left(\omega_{0}\right)$ is the mode-average dielectric permittivity at a carrier frequency $\omega_{0}=\omega\left(k_{0}\right)$, while $\widehat{a}(k)$ is an annihilation operator defined so that

$\left[\widehat{a}\left(k^{\prime}\right), \widehat{a}^{\dagger}(k)\right]=\delta\left(k-k^{\prime}\right)$.

The coefficient $\chi^{(3)}(\mathbf{x})$ is the nonlinear coefficient arising when the electronic polarization field is expanded as a function of the electric displacement, in the commonly used Bloembergen 13 notation (the units are S.I. units, following current standard usage). This may vary along the longitudinal position on the fiber, if the fiber has a variable composition. In terms of modes of the waveguide, and neglecting modal dispersion, the electric displacement field operator $\widehat{\mathbf{D}}(\mathbf{x})$ is:

$\widehat{\mathbf{D}}(\mathbf{x})=i \int d k\left[\frac{\hbar k \epsilon(\omega(k)) v(k)}{4 \pi}\right]^{\frac{1}{2}} \widehat{a}(k) \mathbf{u}(\mathbf{r}) \exp (i k x)+h . c$.

where:

$$
\int d^{2} \mathbf{r}|\mathbf{u}(\mathbf{r})|^{2}=1
$$

Here $v(k)=\partial \omega(k) / \partial k$ is the group velocity. The function $\mathbf{u}(\mathbf{r})$ gives the transverse mode structure. Although a general mode structure can be included, for the purposes of this paper we could equally well assume a square wave-guide of area $A$, which gives $\mathbf{u}(\mathbf{r}) \simeq \mathbf{e}_{y} / \sqrt{A}$. We note here that the above mode expansion for a dispersive medium is a rather general one, and has been worked out both from macroscopic quantization, and from a microscopic model 10 with an arbitrary number of electronic or phonon resonances.

In the infinite volume limit, the polariton field is defined by noting that the annihilation and creation operators can be related to a quantum field using: 
$\widehat{\Psi}(t, x)=\frac{1}{\sqrt{2 \pi}} \int d k \widehat{a}(t, k) \exp \left[i\left(k-k_{0}\right) x+i \omega_{0} t\right]$

This photon-density operator $\widehat{\Psi}(t, x)$ is the slowly varying field annihilation operator for the linear quasi-particle excitations of the coupled electromagnetic and polarization fields traveling through the fiber 8 . The nonzero equal-time commutations relations for these Bose operators are

$$
\left[\widehat{\Psi}(t, x), \widehat{\Psi}^{\dagger}\left(t, x^{\prime}\right)\right]=\delta\left(x-x^{\prime}\right) .
$$

As shown in earlier treatments 2 , the Hamiltonian [Eq. (2.2)] can now be rewritten approximately as:

$\widehat{H}_{F}=\hbar \int d x \int d x^{\prime} \omega\left(x, x^{\prime}\right) \widehat{\Psi}^{\dagger}(t, x) \widehat{\Psi}\left(t, x^{\prime}\right)-\frac{\hbar}{2} \int d x \chi^{E}(x) \widehat{\Psi}^{\dagger 2}(t, x) \widehat{\Psi}^{2}(t, x)$.

Here we have introduced the kernel $\omega\left(x, x^{\prime}\right)$, which is the linear dielectric component of the Hamiltonian, and a nonlinear coupling term $\chi_{E}(x)$. This kernel is then Taylor expanded around $k=k_{0}$, and approximated to quadratic order in $\left(k-k_{0}\right)$, by:

$$
\begin{aligned}
\omega\left(x, x^{\prime}\right) & =\int \frac{d k}{2 \pi} \omega(k) \exp \left[i\left(k-k_{0}\right)\left(x-x^{\prime}\right)\right]-\frac{1}{2} k_{0} v\left(k_{0}\right) \int d^{2} \mathbf{r} \Delta \chi^{(1)}(\mathbf{x})|\mathbf{u}(\mathbf{r})|^{2} \delta\left(x-x^{\prime}\right) \\
& \simeq\left[\omega_{0}+\Delta \omega(x)\right] \delta\left(x-x^{\prime}\right)+\int \frac{d k}{4 \pi}\left[i \omega_{0}^{\prime}\left(\partial_{x^{\prime}}-\partial_{x}\right)+\omega_{0}^{\prime \prime}\left(\partial_{x} \partial_{x^{\prime}}\right)+\cdots\right] \exp \left[i k\left(x-x^{\prime}\right)\right] .
\end{aligned}
$$

In writing down Eq. (2.8), we have assumed that the frequency dependence in the nonlinear coupling can be neglected, which is a good approximation for relatively narrow bandwidths. The nonlinear term is often called the $\chi^{(3)}$ effect, so named because it arises from the third order term in the expansion of the polarization field in terms of the electric field 1 . It causes an electronic contribution $n_{2 e}$ to the intensity dependent refractive index, where: $n=n_{0}+I n_{2}=n_{0}+I\left(n_{2 e}+n_{2 p}\right)$. Thus we define $\chi^{E}$, in units of $[m / s]$, as:

$$
\chi^{E}(x) \equiv\left[\frac{3 \hbar w_{0}^{2} v\left(k_{0}\right)^{2}}{4 \epsilon\left(\omega_{0}\right) c^{2}}\right] \int d^{2} \mathbf{r} \chi^{(3)}(\mathbf{x})|\mathbf{u}(\mathbf{r})|^{4} \equiv\left[\frac{\hbar n_{2 e}(x) \omega_{0}^{2} v^{2}}{A c}\right] .
$$

Here $A=\left[\int d^{2} \mathbf{r}|\mathbf{u}(\mathbf{r})|^{4}\right]^{-1}$ is the effective modal cross-section, and $n_{2 e}$ is the refractive index change per unit field intensity due to electronic transitions. This is less than the total value observed for $n_{2}$, since phonon contributions have yet to be included.

The free evolution part of the total Hamiltonian, which will be removed in subsequent calculations, just describes the carrier frequency $\omega_{0}$. This is not needed in Heisenberg picture calculations for $\widehat{\Psi}(t, x)$, since it is cancelled by the slowly varying field definition. Next, on partial integration of the derivative terms and Fourier transforming, the resulting interaction Hamiltonian $\widehat{H}_{F}^{\prime}$ describing the evolution of $\widehat{\Psi}$ in the slowly varying envelope and rotatingwave approximations is:

$$
\begin{aligned}
\widehat{H}_{F}^{\prime} & =\widehat{H}_{F}-\int d k \hbar \omega_{0} \widehat{a}^{\dagger}(k) \widehat{a}(k) \\
& =\frac{\hbar}{2} \int_{-\infty}^{\infty} d x\left[\Delta \omega(x) \widehat{\Psi}^{\dagger} \widehat{\Psi}+\frac{i v}{2}\left(\nabla \widehat{\Psi}^{\dagger} \widehat{\Psi}-\widehat{\Psi}^{\dagger} \nabla \widehat{\Psi}\right)+\frac{\omega^{\prime \prime}}{2} \nabla \widehat{\Psi}^{\dagger} \nabla \widehat{\Psi}-\frac{\chi^{E}(x)}{2} \widehat{\Psi}^{\dagger 2} \widehat{\Psi}^{2}\right] .
\end{aligned}
$$


For simplicity, only quadratic dispersion is included here. However, the extension to higher-order dispersion is relatively straightforward. This can be achieved by including higher-order terms in the expansion of the dielectric kernel, or else by treating the dispersion as part of the reservoir response function - as in following sections. The response function approach has the advantage that a completely arbitrary polarization response can be included, and transformations to a different frame of reference are simplified. If part of the dielectric response is treated using response functions, then this part of the measured refractive index must be excluded from the free Hamiltonian, to avoid double-counting.

\section{B. Heisenberg equation}

From the interaction Hamiltonian [Eq. 2.11], we find the following Heisenberg equation of motion for the field operator propagating in the $+x$ direction:

$$
\left(v \frac{\partial}{\partial x}+\frac{\partial}{\partial t}\right) \widehat{\Psi}(t, x)=\left[-i \Delta \omega(x)+\frac{i \omega^{\prime \prime}}{2} \frac{\partial^{2}}{\partial x^{2}}+i \chi^{E}(x) \widehat{\Psi}^{\dagger}(t, x) \widehat{\Psi}(t, x)\right] \widehat{\Psi}(t, x)
$$

This is the quantum nonlinear Schrödinger equation in the laboratory frame of reference, which is completely equivalent to the theory of a Bose gas of massive quasi-particles with an effective mass of $\hbar / \omega^{\prime \prime}$ and an average velocity of $v$, for photons near to the carrier frequency of interest. It includes the possibility that the dielectric constant (i.e., the linear response) has a spatial variation, through the term $\Delta \omega(x)$.

We note here that it is occasionally assumed that operators obey equal-space, rather than equal-time commutation relations. This cannot be exactly true, since commutators in an interacting quantum field theory are only well-defined at equal times. At different times, it is possible for a causal effect to propagate to a different spatial location, which can therefore change the unequal-time commutator. In other words, imposing free-field equal-space but unequal-time commutators is not strictly compatible with causality. The assumption of equal-space commutators may be used as an approximation under some circumstances, provided interactions are weak. In this paper, we will use standard equal-time commutators.

\section{Raman Hamiltonian}

To the Hamiltonian given in Eq. (2.11) must be added couplings to linear gain, absorption and phonon reservoirs 16 18. The gain and absorption reservoirs are discussed at length in section 4 . The phonon field consists of thermal and spontaneous excitations in the displacement of atoms from their mean locations in the dielectric lattice. Although previous quantum treatments of Raman scattering have been given 19 , it is necessary to modify these somewhat in the present situation. The Raman interaction energy 20 of a fiber, in terms of atomic displacements from their mean lattice positions, is known to have the form:

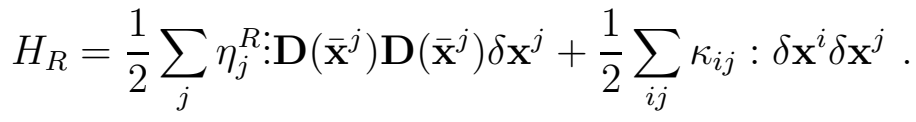

Here $\mathbf{D}\left(\overline{\mathbf{x}}^{j}\right)$ is the electric displacement at the $\mathbf{j}$-th mean atomic location $\overline{\mathbf{x}}^{j}, \delta \mathbf{x}^{j}$ is the atomic displacement operator, $\eta_{j}^{R}$ is a Raman coupling tensor, and $\kappa_{i j}$ represents the short-range atom-atom interactions. 
In order to quantize this interaction with atomic positions using our macroscopic quantization method, we must now take into account the existence of a corresponding set of phonon operators, $\widehat{b}(\omega, x)$ and $\widehat{b}^{\dagger}(\omega, x)$. These operators diagonalize the atomic displacement Hamiltonian in each fiber segment, and have well-defined eigen-frequencies. There are calculations 21 of the frequency spectrum and normal modes of vibration for vitreous silica, using physical models based on the random network theory of disordered systems. The computed vibrational frequency spectrum is remarkably similar to the observed Raman gain profile22. The phonon-photon coupling induces Raman transitions and scattering from acoustic waves (the Brillouin effect) resulting in extra noise sources and an additional contribution to the nonlinearity. The initial state of phonons is thermal, with $n_{t h}(\omega)=[\exp (\hbar \omega / k T)-1]^{-1}$.

\section{A. Hamiltonian and Heisenberg equations}

In terms of these phonon operators, the fiber Hamiltonian in the interaction picture and within the rotating wave approximation for a single polarization is $16 \widehat{H}^{\prime}=\widehat{H}_{R}+\widehat{H}_{F}^{\prime}$, where we have introduced a Raman interaction Hamiltonian:

$\widehat{H}_{R}=\hbar \int_{-\infty}^{\infty} d x \int_{0}^{\infty} d \omega\left\{\widehat{\Psi}^{\dagger}(x) \widehat{\Psi}(x) R(\omega, x)\left[\widehat{b}(\omega, x)+\widehat{b}^{\dagger}(\omega, x)\right]+\omega \widehat{b}^{\dagger}(\omega, x) \widehat{b}(\omega, x)\right\}$.

Here, the atomic vibrations within the silica structure of the fiber are modeled as a continuum of localized oscillators, and are coupled to the radiation modes by a Raman transition with a real frequency dependent coupling $R(\omega, x)$. This coupling could be nonuniform in space, and is determined empirically through measurements of the Raman gain spectrum 16 . The atomic displacement is proportional to $\widehat{b}+\widehat{b}^{\dagger}$, where the phonon annihilation and creation operators, $\widehat{b}$ and $\widehat{b}^{\dagger}$, have the equal-time commutations relations

$$
\left[\widehat{b}(t, \omega, x), \hat{b}^{\dagger}\left(t, \omega^{\prime}, x^{\prime}\right)\right]=\delta\left(x-x^{\prime}\right) \delta\left(\omega-\omega^{\prime}\right) \text {. }
$$

Thus the Raman excitations are treated as an inhomogeneously broadened continuum of modes, localized at each longitudinal location $x$. GAWBS (Guided Wave Acoustic Brillouin Scattering) 2326 is a special case of this, in the low-frequency limit. Since neither Raman nor Brillouin excitations are completely localized, this treatment requires a frequency and wave-number cut-off, so that the field operator $\widehat{\Psi}$ is slowly varying on the phonon scattering distance scale. The corresponding coupled nonlinear operator equations are:

$$
\begin{aligned}
\left(v \frac{\partial}{\partial x}+\frac{\partial}{\partial t}\right) \widehat{\Psi}(t, x) & =i\left[-\Delta \omega(x)+\frac{\omega^{\prime \prime}}{2} \frac{\partial^{2}}{\partial x^{2}}+\chi^{E}(x) \widehat{\Psi}^{\dagger}(t, x) \widehat{\Psi}(t, x)\right] \widehat{\Psi}(t, x) \\
& -i\left\{\int_{0}^{\infty} R(\omega, x)\left[\widehat{b}(t, \omega, x)+\widehat{b}^{\dagger}(t, \omega, x)\right] d \omega\right\} \widehat{\Psi}(t, x) \\
\frac{\partial}{\partial t} \widehat{b}(t, \omega, x) & =-i \omega \widehat{b}(t, \omega, x)-i R(\omega, x) \widehat{\Psi}^{\dagger}(t, x) \widehat{\Psi}(t, x) .
\end{aligned}
$$

In summary, the original theory of nonlinear quantum field propagation is now extended to include both the the electronic and the Raman nonlinearities. The result is a modified Heisenberg equation with a delayed nonlinear response to the field due to the Raman coupling. On integrating the Raman reservoirs, one obtains: 


$$
\left(v \frac{\partial}{\partial x}+\frac{\partial}{\partial t}\right) \widehat{\Psi}(t, x)=i\left[-\Delta \omega(x)+\frac{\omega^{\prime \prime}}{2} \frac{\partial^{2}}{\partial x^{2}}+\int_{0-}^{\infty} d t^{\prime} \chi\left(t^{\prime}, x\right)\left[\widehat{\Psi}^{\dagger} \widehat{\Psi}\right]\left(t-t^{\prime}, x\right)+\widehat{\Gamma}^{R}(t, x)\right] \widehat{\Psi}(t, x),
$$

where

$$
\begin{aligned}
\chi(t, x) & =\chi^{E}(x) \delta(t)+2 \Theta(t) \int_{0}^{\infty} R^{2}(\omega, x) \sin (\omega t) d \omega \\
\widehat{\Gamma}^{R}(t, x) & =-\int_{0}^{\infty} R(\omega, x)\left[\widehat{b}(t, \omega, x)+\widehat{b}^{\dagger}(t, \omega, x)\right] d \omega
\end{aligned}
$$

in which we have defined $\Theta(t)$ as the step function.

The operators $\widehat{\Gamma}^{R}$ and $\widehat{\Gamma}^{R \dagger}$ are stochastic, with Fourier transforms defined using the normal Fourier transform conventions for field operators:

$$
\begin{aligned}
\widehat{\Gamma}^{R}(\omega, x) & =\frac{1}{\sqrt{2 \pi}} \int d t \exp (i \omega t) \widehat{\Gamma}^{R}(t, x) \\
\widehat{\Gamma}^{R \dagger}(\omega, x) & =\frac{1}{\sqrt{2 \pi}} \int d t \exp (-i \omega t) \widehat{\Gamma}^{R}(t, x) .
\end{aligned}
$$

The frequency-space correlations are given by:

$$
\left\langle\widehat{\Gamma}^{R \dagger}\left(\omega^{\prime}, x^{\prime}\right) \widehat{\Gamma}^{R}(\omega, x)\right\rangle=2 \chi^{\prime \prime}(x,|\omega|)\left[n_{t h}(|\omega|)+\Theta(-\omega)\right] \delta\left(x-x^{\prime}\right) \delta\left(\omega-\omega^{\prime}\right) .
$$

In this expression, we introduce a Raman amplitude gain of $\chi^{\prime \prime}$ per unit photon flux, equal to the imaginary part of the Fourier transform of $\chi(t, x)$, so that: $\chi^{\prime \prime}(x,|\omega|)=\pi R^{2}(x,|\omega|)$. Here we use the Bloembergen normalization for response function Fourier transforms,

$\widetilde{\chi}(\omega, x)=\int d t \exp (i \omega t) \chi(t, x)$,

which does not have the $\sqrt{2 \pi}$ factor included.

Of some significance is the physical interpretation of the correlation functions, which can be regarded as directly contributing to the normally ordered spectrum of the transmitted field. Given a cw carrier, the correlations when $\omega$ is positive correspond to an anti-Stokes (blue-shifted) spectral term, which is clearly zero unless the thermal phonon population is appreciable. However, when $\omega$ is negative, the theta function term indicates that the Stokes (red-shifted) spectral term is nonzero, due to spontaneous Stokes photons emitted even at zero temperature.

\section{B. Raman gain measurements}

The measured intensity gain due to Raman effects at a given relative frequency $\omega$ per unit length, per unit carrier photon flux $I_{0}=v\left\langle\widehat{\Psi}^{\dagger} \widehat{\Psi}\right\rangle$, is:

$$
\frac{1}{I_{0}} \frac{\partial \ln I}{\partial x}=-2 \chi^{\prime \prime}(\omega, x) / v^{2}
$$

Here the gain is positive for Stokes-shifted frequencies $(\omega<0)$, and negative for anti-Stokes $(\omega>0)$, as one would expect. This relationship allows the coupling to be estimated from 
measured Raman gain and fluorescence properties. The simplest way to achieve this goal is to expand the Raman response function in terms of a multiple-Lorentzian model, which can then be fitted to observed Raman fluorescence data using a nonlinear least-squares fit. We therefore expand:

$\chi(t, x)=\chi^{E}(x) \delta(t)+\chi(x) \Theta(t) \sum_{j=0}^{n} F_{j} \delta_{j} \exp \left(-\delta_{j} t\right) \sin \left(\omega_{j} t\right)$.

For normalization purposes, we have introduced $\chi(x)$, which is defined as the total effective nonlinear phase-shift coefficient per unit time and photon density (in units of $\mathrm{rad} . \mathrm{m} / \mathrm{s}$ ), obtained from the low-frequency nonlinear refractive index. This is given in terms of the electronic or fast-responding nonlinear coefficient, $\chi^{E}(x)$, together with the Raman contribution, by integrating over time:

$\chi(x)=\chi^{E}(x)+2 \int_{0}^{\infty} \int_{0}^{\infty} R^{2}(\omega, x) \sin (\omega t) d \omega d t$.

In the above expansion, $F_{j}$ are a set of dimensionless Lorentzian strengths, and $\omega_{j}$ and $\delta_{j}$ are the resonant frequencies and widths respectively, of the effective Raman resonances at each frequency. To improve convergence, the Lorentzian strength parameters are not constrained to be positive. The $j=0$ Lorentzian models the Brillouin contribution to the response function. In general, all of these parameters could be $x$-dependent, but we will often assume that they are constant in space for notational simplicity. The values for an $n=10$ fit in the case of a typical fused silica fiber are given in Table 1, including an estimate of the effective Brillouin contribution averaged over the individual Brillouin scattering peaks. The coefficient of the electronic nonlinearity is now obtained explicitly in terms of the total nonlinear refractive index:

$\chi^{E}(x)=\frac{\hbar(1-f) n_{2} \omega_{0}^{2} v^{2}}{A c}$

where $\omega_{0}$ is the carrier frequency, $A$ is the effective cross-sectional area of the traveling mode, and $f$ is the fraction of the nonlinearity due to the Raman gain, which has been estimated using the procedure outlined above:

$$
\begin{aligned}
f & =\frac{\chi^{R}}{\chi}=\frac{2}{\chi} \int_{0}^{\infty} d t \int_{0}^{\infty} d \omega R^{2}(\omega, x) \sin (\omega t) \\
& \simeq 0.2 .
\end{aligned}
$$

A result of this model is that the phonon operators do not have white noise behaviour. In fact, this colored noise property is significant enough to invalidate the usual Markovian and rotating-wave approximations, which are therefore not used in the phonon equations. Of course, the photon modes may also be in a thermal state of some type. However, thermal effects are typically much more important at the low frequencies that characterize Raman and Brillouin scattering, than they are at optical frequencies. In addition, if the input is a photon field generated by a laser, any departures from coherent statistics will be rather specific to the laser type, instead of having the generic properties of thermal fields.

Finally, there is another effect which has been so far neglected. This is the ultra-low frequency tunneling due to lattice defects27. As this is not strictly linear, it can not be 
included accurately in our macroscopic Hamiltonian. Despite this, the effects of this $1 / f$ type noise may be included approximately for any predetermined temperature. This can be achieved by simply modifying the refractive index perturbation term so that it becomes $\Delta \omega(t, x)$ and generates the known low-frequency refractive index fluctuations.

\section{Gain and absorption}

In silica optical fibers, there is a relatively flat absorption profile, with a minimum absorption coefficient of approximately $0.2 \mathrm{~dB} / \mathrm{km}$ in the vicinity of the commonly used communications wavelengths of around $\lambda=1.5 \mu \mathrm{m}$. This effect can be compensated for by the use of fiber laser amplifiers, resulting in nearly zero net absorption over a total link that includes both normal and amplified fiber segments. In practical terms, this situation leads to an approximately uniform fiber environment, provided the net gain and loss are spatially modulated more rapidly than than the pulse dispersion length. These additional effects need to be included within the present Hamiltonian model, in order to have a fully consistent quantum theory. For wide-band communications systems, either with time-domain multiplexing or frequencydomain multiplexing, it can become necessary to include the frequency-dependence and spatial variation of the gain and loss terms. This is especially true if spectral filters are included in the fiber.

\section{A. Absorbing reservoirs}

The absorption reservoir is modeled most simply by a coupling to a continuum of harmonic oscillators at resonant frequency $\omega$. In the interaction picture used here, the Hamiltonian term causing rapidly varying operator evolution of the reservoir at the carrier frequency $\omega_{0}$ is subtracted, leaving:

$\widehat{H}_{A}^{\prime}=\hbar \int_{-\infty}^{\infty} d x \int_{0}^{\infty} d \omega\left\{\left[\widehat{\Psi}(x) \widehat{a}^{\dagger}(\omega, x) A(\omega, x)+\right.\right.$ h.c. $\left.]+\left(\omega-\omega_{0}\right) \widehat{a}^{\dagger} \widehat{a}(\omega, x)\right\}$,

where $A(\omega, x)$ provides the frequency dependent coupling between the radiation modes and the absorption reservoirs. The reservoir annihilation and creation operators, $\widehat{a}$ and $\widehat{a}^{\dagger}$, have the commutation relations

$$
\left[\widehat{a}(\omega, x), \widehat{a}^{\dagger}\left(\omega^{\prime}, x^{\prime}\right)\right]=\delta\left(x-x^{\prime}\right) \delta\left(\omega-\omega^{\prime}\right) .
$$

The equations for the absorbing photon reservoirs can be integrated immediately. The photon reservoir variable, for instance, obeys:

$\frac{\partial}{\partial t} \widehat{a}(t, \omega, x)=-i\left(\omega-\omega_{0}\right) \widehat{a}(t, \omega, x)-i A(\omega, x) \widehat{\Psi}(t, x)$.

Hence, the solutions are:

$\widehat{a}(t, \omega, x)=\widehat{a}\left(t_{0}, \omega, x\right) \exp \left[-i\left(\omega-\omega_{0}\right)\left(t-t_{0}\right)\right]-i A(\omega, x) \int_{t_{0}}^{t} \exp \left[-i\left(\omega-\omega_{0}\right)\left(t-t^{\prime}\right)\right] \widehat{\Psi}\left(t^{\prime}, x\right) d t^{\prime}$, 
with initial correlations for the reservoir variables in the far past $\left(t_{0} \rightarrow-\infty\right)$ given by:

$$
\begin{aligned}
\left\langle\widehat{a}^{\dagger}\left(t_{0}, \omega, x\right) \widehat{a}\left(t_{0}, \omega^{\prime}, x^{\prime}\right)\right\rangle & =n_{t h}(\omega) \delta\left(x-x^{\prime}\right) \delta\left(\omega-\omega^{\prime}\right), \\
\left\langle\widehat{a}\left(t_{0}, \omega, x\right) \widehat{a}^{\dagger}\left(t_{0}, \omega^{\prime}, x^{\prime}\right)\right\rangle & =\left[n_{t h}(\omega)+1\right] \delta\left(x-x^{\prime}\right) \delta\left(\omega-\omega^{\prime}\right) .
\end{aligned}
$$

The solution for $\widehat{a}(t, \omega, x)$ is substituted into the Heisenberg equation for the field evolution, giving rise to an extra time-dependent term, of the form:

$$
\begin{aligned}
-i \int_{0}^{\infty} A^{*}(\omega, x) \widehat{a}(t, \omega, x) d \omega & =-\int_{0}^{\infty} d \omega|A(\omega, x)|^{2} \int_{t_{0}}^{t} d t^{\prime} \exp \left[-i\left(\omega-\omega_{0}\right)\left(t-t^{\prime}\right)\right] \widehat{\Psi}\left(t^{\prime}, x\right) \\
& -i \int_{0}^{\infty} d \omega A^{*}(\omega, x) \exp \left[-i\left(\omega-\omega_{0}\right)\left(t-t_{0}\right)\right] \widehat{a}\left(t_{0}, \omega, x\right) \\
& =-\int_{0}^{\infty} d t^{\prime \prime} \gamma^{A}\left(t^{\prime \prime}, x\right) \widehat{\Psi}\left(t-t^{\prime \prime}, x\right)+\widehat{\Gamma}^{A}(t, x),
\end{aligned}
$$

where $t^{\prime \prime}=t-t^{\prime}$, and the response function and reservoir terms are obtained most simply by extending the lower limit on the frequency integral to $-\infty$, introducing only an infinitesimal error in the process, so that:

$$
\begin{aligned}
& \gamma^{A}(t, x) \approx \Theta(t) \int_{-\infty}^{+\infty} d \omega|A(\omega, x)|^{2} \exp \left[-i\left(\omega-\omega_{0}\right) t\right] \\
& \widehat{\Gamma}^{A}(t, x)=-i \int_{0}^{\infty} d \omega A^{\dagger}(\omega, x) \exp \left[-i\left(\omega-\omega_{0}\right)\left(t-t_{0}\right)\right] \widehat{a}\left(t_{0}, \omega, x\right) .
\end{aligned}
$$

The response function integral represents a deterministic or 'drift' term to the motion, with a Fourier transform of:

$\widetilde{\gamma}^{A}(\omega, x)=\int \gamma^{A}(t, x) \exp (i \omega t) d t=\gamma^{A}(\omega, x)+i \gamma^{A \prime \prime}(\omega, x)$

so that the amplitude loss rate is:

$\gamma^{A}(\omega, x)=\pi\left|A\left(\omega_{0}+\omega, x\right)\right|^{2}$.

In the case of a spatially uniform reservoir with a flat spectral density, the Wigner-Weiskopff approximation (neglecting frequency shifts) gives a uniform Markovian loss term with:

$\gamma^{A}(t) \approx \widetilde{\gamma}^{A} \delta(t)$

where the average amplitude loss coefficient is:

$$
\begin{aligned}
\widetilde{\gamma}^{A}=\widetilde{\gamma}^{A}(0) & =\int_{0}^{\infty} \int_{-\infty}^{+\infty} d t d \omega|A(\omega)|^{2} \exp \left[-i\left(\omega-\omega_{0}\right) t\right] \\
& =\gamma^{A}+i \gamma^{A \prime \prime}
\end{aligned}
$$

This approximation, known as the Markov approximation, is generally rather accurate for the absorbing reservoirs, whose response does not typically vary fast with frequency. An exception to this rule would be any case involving resonant impurities in the fiber, or very short pulses whose bandwidth is comparable to the frequency-scale of absorption changes. 
The second quantity in Eq. (4.7), $\widehat{\Gamma}^{A}(t, x)$, behaves like a stochastic term due to the random initial conditions. Neglecting the frequency dependence of the thermal photon number, the corresponding correlation functions are

$$
\begin{aligned}
\left\langle\widehat{\Gamma}^{A}(t, x) \widehat{\Gamma}^{A \dagger}\left(t^{\prime}, x^{\prime}\right)\right\rangle & =\int_{0}^{\infty} d \omega|A(\omega, x)|^{2} \exp \left[-i\left(\omega-\omega_{0}\right)\left(t-t^{\prime}\right)\right]\left[n_{t h}(\omega)+1\right] \delta\left(x-x^{\prime}\right) \\
& \approx\left[\gamma^{A}\left(t-t^{\prime}, x\right)+\gamma^{A *}\left(t^{\prime}-t, x\right)\right]\left[n_{t h}\left(\omega_{0}\right)+1\right] \delta\left(x-x^{\prime}\right)
\end{aligned}
$$

and:

$$
\begin{aligned}
\left\langle\widehat{\Gamma}^{A \dagger}\left(t^{\prime}, x^{\prime}\right) \widehat{\Gamma}^{A}(t, x)\right\rangle & =\int_{0}^{\infty} d \omega|A(\omega, x)|^{2} \exp \left[-i\left(\omega-\omega_{0}\right)\left(t-t^{\prime}\right)\right] n_{t h}(\omega) \delta\left(x-x^{\prime}\right) \\
& \approx\left[\gamma^{A}\left(t-t^{\prime}, x\right)+\gamma^{A *}\left(t^{\prime}-t, x\right)\right] n_{t h}\left(\omega_{0}\right) \delta\left(x-x^{\prime}\right) .
\end{aligned}
$$

At optical or infra-red frequencies, it is a good approximation to set $n_{t h}\left(\omega_{0}\right)=0$. On Fourier transforming the noise sources, one then obtains:

$$
\left\langle\widehat{\Gamma}^{A}(\omega, x) \widehat{\Gamma}^{A \dagger}\left(\omega^{\prime}, x^{\prime}\right)\right\rangle=2 \gamma^{A}(\omega, x) \delta\left(x-x^{\prime}\right) \delta\left(\omega-\omega^{\prime}\right) .
$$

Again taking the simplifying case of a spatially uniform reservoir in the Wigner-Weiskopff limit, this reduces to:

$$
\begin{aligned}
\left\langle\widehat{\Gamma}^{A}(t, x) \widehat{\Gamma}^{A \dagger}\left(t^{\prime}, x^{\prime}\right)\right\rangle & =2 \gamma^{A} \delta\left(t-t^{\prime}\right) \delta\left(x-x^{\prime}\right) \\
\left\langle\widehat{\Gamma}^{A \dagger}(t, x) \widehat{\Gamma}^{A}\left(t^{\prime}, x^{\prime}\right)\right\rangle & =0 .
\end{aligned}
$$

Note that the dimensions for the amplitude relaxation rates are $\left[\gamma^{A}\right]=s^{-1}$. It is easy to show that $2 \gamma^{A} / v$ corresponds to the usual linear absorption coefficient for fibers during propagation. A typical measured absorption figure in current fused silica communications fibers is $0.2 \mathrm{~dB} / \mathrm{km}$ in the minimum region of absorption (near $\lambda=1.5 \mu \mathrm{m}$ ). The corresponding absorption coefficient is $2 \gamma^{A} / v \simeq 2.3 \times 10^{-5} \mathrm{~m}^{-1}$. To the extent that this effect is wavelength (and hence frequency) dependent, the resulting dispersion can be included as well, giving rise to a complete response function $\gamma^{A}(t)$ for absorption. Non-Markovian effects like this can either be neglected completely - which is a good approximation for slowly varying absorption in undoped fiber - or else included in the correlation functions of the reservoirs as given above.

The physical meaning of the reservoir operator spectral correlations is best understood by considering the effect of these terms on photodetection, which according to photodetection theory, means a normally ordered field correlation. This involves normally ordered reservoir correlations to lowest order. Since these are zero, we conclude that the absorbing reservoirs essentially add no quantum noise that is observable via normal photodetection methods.

\section{B. Waveguide laser amplifiers}

The equations for gain or laser reservoirs are generally more complex, involving the nonlinear response of atomic impurities added to provide some gain in the fiber medium. This also involves a pump process (usually from a semiconductor laser) to maintain the lasing atoms in an inverted state. In the case of silica fibers, a commonly used lasing transition is provided 
by erbium impurities 28 . The effect of these gain reservoirs is typically to introduce new types of dispersion, owing to the frequency dependence of the gain 29 . In addition, there are new nonlinear effects, due to the effects of saturation - which in turn depend on the pumping intensity.

It is possible to develop a detailed theory of erbium laser amplifiers. However, this paper will treat the simplest possible quantum theory of a traveling-wave quantum-limited laser amplifier. More details of the quantum theory, including nonlinear effects, are treated elsewhere30. However, the simple theory presented here provides a microscopic justification for observed quantum noise effects in fiber amplifier chains. In particular, it reproduces the results of the phenomenological theory of Gordon and Haust, which is known to give predictions in accord with soliton transmission experiments. The resulting "Gordon-Haus jitter" can be reduced through the use of filtering techniques. Assuming that the laser amplifier is polarization-insensitive, we again omit the polarization index. The reservoir variable $\widehat{\sigma}_{\mu}=|1\rangle_{\mu}\left\langle\left. 2\right|_{\mu}\right.$ is an atomic transition operator, which induces a near-resonant atomic transition from an upper to a lower state, with two-level transitions having an assumed density of $\rho(\omega, x)$ in position and resonant angular frequency $\omega$.

These quantum effects are modeled here by including gain reservoirs in the Hamiltonian, coupled by a frequency dependent term $G(\omega, x)$ to the radiating field. Here the gain terms $\widehat{\sigma}^{ \pm}(\omega, x, t)$ represent the raising and lowering Pauli field operators, for two-level lasing transitions at frequency $\omega$. In more detail, we have gain given by an interaction Hamiltonian:

$\widehat{H}_{G}^{\prime}=\hbar \int_{-\infty}^{\infty} d x \int_{0}^{\infty} d \omega\left\{\left[\widehat{\Psi} \widehat{\sigma}^{+}(\omega, x) G(\omega, x)+h . c.\right]+\frac{\omega-\omega_{0}}{2} \sigma^{z}(\omega, x)\right\}$,

where the atomic raising and lowering field operators, $\widehat{\sigma}^{ \pm}$, are defined in terms of discrete Pauli operators, by:

$$
\begin{aligned}
\widehat{\sigma}^{+}(\omega, x, t) & =\frac{1}{\sqrt{\rho(\omega, x)}} \sum_{\mu}|2\rangle\left\langle\left. 1\right|_{\mu} \exp \left(-i \omega_{0} t\right) \delta\left(x-x_{\mu}\right) \delta\left(\omega-\omega_{\mu}\right),\right. \\
\hat{\sigma}^{-}(\omega, x, t) & =\frac{1}{\sqrt{\rho(\omega, x)}} \sum_{\mu}|1\rangle\left\langle\left. 2\right|_{\mu} \exp \left(i \omega_{0} t\right) \delta\left(x-x_{\mu}\right) \delta\left(\omega-\omega_{\mu}\right),\right. \\
\hat{\sigma}^{z}(\omega, x, t) & =\frac{1}{\rho(\omega, x)} \sum_{\mu}[|2\rangle\langle 2|-| 1\rangle\langle 1|]_{\mu} \delta\left(x-x_{\mu}\right) \delta\left(\omega-\omega_{\mu}\right) .
\end{aligned}
$$

These operators are in general time-dependent in the Heisenberg picture, but have the equal-time commutation relations:

$$
\left[\widehat{\sigma}^{+}(t, \omega, x), \widehat{\sigma}^{-}\left(t, \omega^{\prime}, x^{\prime}\right)\right]=\widehat{\sigma}^{z}(t, \omega, x) \delta\left(x-x^{\prime}\right) \delta\left(\omega-\omega^{\prime}\right) .
$$

In the limit of complete inversion, with linear response and pure inhomogeneous broadening,

$$
\frac{\partial}{\partial t} \widehat{\sigma}^{-}(t, \omega, x)=-i\left(\omega-\omega_{0}\right) \widehat{\sigma}^{-}(t, \omega, x)+i \widehat{\sigma}^{z}(t, \omega, x) G(\omega, x) \widehat{\Psi}(t, x) .
$$

Hence, the solutions in the amplifier case are:

$$
\begin{aligned}
\widehat{\sigma}^{-}(t, \omega, x) & =\widehat{\sigma}^{-}\left(t_{0}, \omega, x\right) \exp \left[-i\left(\omega-\omega_{0}\right)\left(t-t_{0}\right)\right] \\
& +i G(\omega, x) \int_{t_{0}}^{t} \exp \left[-i\left(\omega-\omega_{0}\right)\left(t-t^{\prime}\right)\right] \widehat{\sigma}^{z}\left(t^{\prime} \omega, x\right) \widehat{\Psi}\left(t^{\prime}, x\right) d t^{\prime}
\end{aligned}
$$


With complete inversion, $\left\langle\widehat{\sigma}^{z}\left(t_{0}, \omega, x\right)\right\rangle=1$, so the initial correlations for the reservoir variables in the far past $\left(t_{0} \rightarrow-\infty\right)$ are given by:

$\left\langle\widehat{\sigma}^{+}\left(t_{0}, \omega, x\right) \widehat{\sigma}^{-}\left(t_{0}, \omega^{\prime}, x^{\prime}\right)\right\rangle=\delta\left(x-x^{\prime}\right) \delta\left(\omega-\omega^{\prime}\right)$,
$\left\langle\widehat{\sigma}^{-}\left(t_{0}, \omega, x\right) \widehat{\sigma}^{+}\left(t_{0}, \omega^{\prime}, x^{\prime}\right)\right\rangle=0$.

We substitute the solution for $\widehat{\sigma}^{-}(t, \omega, x)$ into the Heisenberg equation for the field evolution, assuming no depletion of the inversion, and trace over the atomic gain reservoirs. This gives rise to an extra time-dependent term, of the form:

$$
\begin{aligned}
-i \int_{0}^{\infty} G^{*}(\omega, x) \widehat{\sigma}^{-}(t, \omega, x) d \omega & =\int_{0}^{\infty} d \omega|G(\omega, x)|^{2} \int_{t_{0}}^{t} d t^{\prime} \exp \left[-i\left(\omega-\omega_{0}\right)\left(t-t^{\prime}\right)\right] \widehat{\Psi}\left(t^{\prime}, x\right) \\
& -i \int_{0}^{\infty} d \omega G^{*}(\omega, x) \exp \left[-i\left(\omega-\omega_{0}\right)\left(t-t_{0}\right)\right] \sigma^{-}\left(t_{0}, \omega, x\right) \\
& =\int_{0}^{\infty} d t^{\prime \prime} \gamma^{G}\left(t^{\prime \prime}, x\right) \widehat{\Psi}\left(t-t^{\prime \prime}, x\right)+\widehat{\Gamma}^{G}(t, x),
\end{aligned}
$$

where $t^{\prime \prime}=t-t^{\prime}$, as before. This gives:

$$
\begin{aligned}
& \gamma^{G}(t, x) \approx \Theta(t) \int_{-\infty}^{+\infty} d \omega|G(\omega, x)|^{2} \exp \left[-i\left(\omega-\omega_{0}\right) t\right] \\
& \widehat{\Gamma}^{G}(t, x) \approx-i \int_{-\infty}^{\infty} d \omega G^{*}(\omega, x) \exp \left[-i\left(\omega-\omega_{0}\right)\left(t-t_{0}\right)\right] \sigma^{-}\left(t_{0}, \omega, x\right) .
\end{aligned}
$$

Fourier transforming the response function gives:

$\widetilde{\gamma}^{G}(\omega, x)=\int \gamma^{G}(t, x) \exp (i \omega t) d t=\gamma^{G}(\omega, x)+i \gamma^{G \prime \prime}(\omega, x)$

and the (real) resonant amplitude gain coefficient is:

$\gamma^{G}(\omega, x)=\pi\left|G\left(\omega+\omega_{0}, x\right)\right|^{2}$.

As with the loss case, $\widehat{\Gamma}^{G}(t, x)$ behaves like a stochastic term due to the random initial conditions. The corresponding correlation functions are

$$
\begin{aligned}
\left\langle\widehat{\Gamma}^{G \dagger}\left(t^{\prime}, x^{\prime}\right) \widehat{\Gamma}^{G}(t, x)\right\rangle & =\int_{0}^{\infty} d \omega|G(\omega, x)|^{2} \exp \left[i\left(\omega-\omega_{0}\right)\left(t-t^{\prime}\right)\right] \delta\left(x-x^{\prime}\right) \\
& =\left[\gamma^{G}\left(t-t^{\prime}, x\right)+\gamma^{G^{*}}\left(t^{\prime}-t, x\right)\right] \delta\left(x-x^{\prime}\right)
\end{aligned}
$$

Fourier transforming these noise sources gives:

$\left\langle\widehat{\Gamma}^{G \dagger}\left(\omega^{\prime}, x^{\prime}\right) \widehat{\Gamma}^{G}(\omega, x)\right\rangle=2 \gamma^{G}(\omega, x) \delta\left(x-x^{\prime}\right) \delta\left(\omega-\omega^{\prime}\right)$

Taking the uniform fiber in the Wigner-Weiskopff limit as before, so $\gamma^{G}=\gamma^{G}(0, x)$, this reduces to:

$$
\begin{aligned}
\left\langle\widehat{\Gamma}^{G}(t, x) \widehat{\Gamma}^{G \dagger}\left(t^{\prime}, x^{\prime}\right)\right\rangle & =0 \\
\left\langle\widehat{\Gamma}^{G \dagger}(t, x) \widehat{\Gamma}^{G}\left(t^{\prime}, x^{\prime}\right)\right\rangle & =2 \gamma^{G} \delta\left(t-t^{\prime}\right) \delta\left(x-x^{\prime}\right) .
\end{aligned}
$$


The dimensions for the amplitude gain are $\left[\gamma^{G}\right]=s^{-1}$. On Fourier transforming, the response function can be related to the measured intensity gain $2 \operatorname{Re}\left[\widetilde{\gamma}^{G}(\omega, x) / v\right]$ at any frequency offset $\omega$, relative to the carrier frequency $\omega_{0}$. This allows one to obtain the linear gain coefficient for fibers during propagation. Since measured laser gain figures can be much greater than the absorption, it is possible to compensate for fiber absorption with relatively short regions of gain. The results presented here are only valid in the linear gain regime. More generally, a functional Taylor expansion up to at least third order in the field would be needed to represent the full nonlinear response of the laser amplifier, together with additional quantum noise terms.

Finally, it is necessary to consider the result of incomplete inversion of an amplifier. Here, the noninverted atoms give rise to absorption, not gain, and will generate additional quantum-noise and absorption response terms. These must be treated as in the previous section, including non-Markovian effects if the absorption line is narrow-band. An important consequence is that the measured gain only gives the difference between the gain and the loss. This doesn't cause any problems with the deterministic response function - but it does cause difficulties in determining the amplifier quantum noise levels, which can only be uniquely determined through spontaneous fluorescence measurements. Obviously, the lowest quantum noise levels occur when all the lasing transitions are completely inverted.

The physical meaning of the reservoir operator spectral correlations for the amplifier case is clearly quite different to the case of the absorber. If we consider the effect of these terms on photodetection as before, which means a normally ordered field correlation, we should look again at the normally ordered correlations of the reservoirs. Since these are no longer zero, we conclude that the amplifying reservoirs emit fluorescent photons due to spontaneous emission over the amplifier bandwidth.

\section{Combined Heisenberg Equations}

Coupling linear gain and absorption reservoirs in this way to the Raman-modified Heisenberg equation leads to a generalized quantum nonlinear Schrödinger equation. Such equations are sometimes called quantum Langevin equations. In the present case of a single polarization, the resulting field equations are:

$$
\begin{aligned}
\left(v \frac{\partial}{\partial x}+\frac{\partial}{\partial t}\right) \widehat{\Psi}(t, x) & =-\int_{0}^{\infty} d t^{\prime} \gamma\left(t^{\prime}, x\right) \widehat{\Psi}\left(t-t^{\prime}, x\right)+\widehat{\Gamma}(t, x) \\
& +i\left[\frac{\omega^{\prime \prime}}{2} \frac{\partial^{2}}{\partial x^{2}}+\int_{0-}^{\infty} d t^{\prime} \chi\left(t^{\prime}\right)\left[\widehat{\Psi}^{\dagger} \widehat{\Psi}\right]\left(t-t^{\prime}, x\right)+\widehat{\Gamma}^{R}(t, x)\right] \widehat{\Psi}(t, x) .
\end{aligned}
$$

In this equation,

$\gamma(t, x)=\gamma^{A}(t, x)-\gamma^{G}(t, x)+i \Delta \omega(x) \delta(t)$

is a net linear response function due to a coupling to linear gain/absorption reservoirs, including the effects of a spatially varying refractive index. This can be Fourier transformed, giving: $\widetilde{\gamma}(\omega, x)=\gamma(\omega, x)+i \gamma^{\prime \prime}(\omega, x)$, where $\gamma(\omega, x)<0$ for gain, and $\gamma(\omega, x)>0$ for absorption. Similarly, $\widehat{\Gamma}(t, x)$ is the linear quantum noise due to gain and absorption. The actual measured intensity gain at frequency $\omega+\omega_{0}$ is given in units of $\left[\mathrm{m}^{-1}\right]$, by: 


$$
\frac{\partial \ln I}{\partial x}=2\left(\gamma^{G}(\omega, x)-\gamma^{A}(\omega, x)\right) / v .
$$

The stochastic terms have the correlations

$$
\begin{aligned}
\left\langle\widehat{\Gamma}^{R \dagger}\left(\omega^{\prime}, x^{\prime}\right) \widehat{\Gamma}^{R}(\omega, x)\right\rangle & =2 \chi^{\prime \prime}(x,|\omega|)\left[n_{t h}(|\omega|)+\Theta(-\omega)\right] \delta\left(x-x^{\prime}\right) \delta\left(\omega-\omega^{\prime}\right) \\
\left\langle\widehat{\Gamma}^{\dagger}\left(\omega^{\prime}, x^{\prime}\right) \widehat{\Gamma}(\omega, x)\right\rangle & =2 \gamma^{G}(\omega, x) \delta\left(x-x^{\prime}\right) \delta\left(\omega-\omega^{\prime}\right) \\
\left\langle\widehat{\Gamma}(\omega, x) \widehat{\Gamma}^{\dagger}\left(\omega^{\prime}, x^{\prime}\right)\right\rangle & =2 \gamma^{A}(\omega, x) \delta\left(x-x^{\prime}\right) \delta\left(\omega-\omega^{\prime}\right)
\end{aligned}
$$

where we have introduced minimal linear quantum noise terms $\Gamma$ and $\Gamma^{\dagger}$ for the gain/absorption reservoirs, and where thermal photons have been neglected (since usually $\hbar \omega_{0}>>k T$, as explained in section 4). Equation (5.1) can be easily generalized to include nonlinear absorption or laser saturation effects, relevant to amplifiers with intense fields, but these terms are omitted here for simplicity.

This complete Heisenberg equation gives a consistent quantum theoretical description of dispersion, nonlinear refractive index, Raman/GAWBS scattering, linear gain, and absorption. It is important to notice that the reservoir correlations have a simple physical interpretation, especially in the zero-temperature limit. Normally ordered noise correlations occur when there is gain, anti-normally ordered correlations when there is absorption. This is the reason why the normally ordered Raman noise correlations vanish at zero temperature for positive frequencies. At low temperatures, Raman processes only cause absorption to occur at positive detunings from a pump frequency. Thermal correlations have a more classical behaviour, and occur for both types of operator ordering.

It is often useful to do calculations in a simpler model, in which we include the effects of uniform gain and loss in a moving frame. This can either be carried out using a standard moving frame $\left(x_{v}=x-v t\right)$, or with a propagative time $\left(t_{v}=t-x / v\right)$ as in the original Gordon-Haus calculations. For propagative calculations, it is most convenient to use photon flux operators

$\widehat{\Phi}\left(t_{v}, x\right)=\sqrt{v} \widehat{\Psi}(t, x)$.

For long pulses, assuming a uniform gain/loss response in the frequency domain, the propagative transformation gives the following approximate equations:

$$
\begin{aligned}
\frac{\partial}{\partial x} \widehat{\Phi}\left(t_{v}, x\right) & =-\int_{0}^{\infty} d t_{v}^{\prime} \frac{\gamma\left(t_{v}^{\prime}, x\right)}{v} \widehat{\Phi}\left(t_{v}-t_{v}^{\prime}, x\right)+\widehat{\Gamma}(t) / \sqrt{v} \\
& +i\left[-\frac{k^{\prime \prime}}{2} \frac{\partial^{2}}{\partial t_{v}{ }^{2}}+\int_{0-}^{\infty} d t^{\prime} \frac{\chi\left(t_{v}^{\prime}\right)}{v^{2}}\left[\widehat{\Phi}^{\dagger} \widehat{\Phi}\right]\left(t_{v}-t_{v}^{\prime}, x\right)+\frac{1}{v} \widehat{\Gamma}^{R}\right] \widehat{\Phi}\left(t_{v}, x\right)
\end{aligned}
$$

In addition, if the pulses are narrow-band compared to the gain and loss bandwidths, and the reservoirs are uniform, then the gain and absorption reservoirs are nearly delta-correlated, with

$$
\begin{aligned}
\left\langle\widehat{\Gamma}^{\dagger}\left(t, x_{v}\right) \widehat{\Gamma}\left(t^{\prime}, x_{v}^{\prime}\right)\right\rangle & =2 \gamma^{G} \delta\left(x_{v}-x_{v}{ }^{\prime}\right) \delta\left(t-t^{\prime}\right) \\
\left\langle\widehat{\Gamma}\left(t, x_{v}\right) \widehat{\Gamma}^{\dagger}\left(t^{\prime}, x_{v}^{\prime}\right)\right\rangle & =2 \gamma^{A} \delta\left(x_{v}-x_{v}{ }^{\prime}\right) \delta\left(t-t^{\prime}\right) .
\end{aligned}
$$

It is essentially this set of approximate equations that corresponds to those used to predict the soliton 31 self-frequency shift 32 and related effectst in soliton propagation, except for the omission of the Raman reservoir terms. 


\section{Phase-space methods}

The Heisenberg equations are not readily soluble in their present form. To generate numerical equations for analytic calculations or for simulation, operator representation theory can be used. There is more than one possible method, depending on which phasespace representation is used. The positive- $P$ representation, for example, produces exact results 1 16 6 provided phase-space boundary terms are negligible, while a truncated Wigner representation 33 . 34 gives approximate results that are valid in the limit of large photon number. It is important to note that the Wigner method represents symmetrically ordered rather than normally ordered operator products, and so has finite quantum noise terms even for a vacuum field. These can be thought of as corresponding to the shot noise detected in a homodyne or local-oscillator measurement, while the positive- $P$ representation represents normally ordered operators, and therefore corresponds to direct-detection noise.

Either technique can be used for this problem, each with its characteristic advantages and disadvantages. The positive- $P$ representation, although exact, uses an enlarged phase-space which therefore takes longer to simulate numerically. It only includes normally ordered noise and initial conditions, and this is an advantage in some cases, since the resulting noise is zero in the vacuum state. The Wigner technique is simpler, and for large mode occupations, its results are accurate enough for many purposes. However, it has the drawback that it includes symmetrically ordered vacuum fluctuations.

First, we expand the field operators in terms of operators for the free-field modes. Applying the appropriate operator correspondences to the master equation for the reduced density operator $\hat{\rho}_{\Psi}$ in which the reservoir modes have been traced over, namely

$\widehat{\dot{\rho}}_{\Psi}=\operatorname{Tr}_{R} \widehat{\dot{\rho}}=\operatorname{Tr}_{R} \frac{1}{i \hbar}[\widehat{H}, \widehat{\rho}]$,

gives a functional equation for the corresponding operator representation.

In the positive- $P$ case, the equation is defined on a functional phase-space of double the classical dimensions, so that a complete expansion in terms of a coherent-state basis $|\Psi\rangle$ is obtained:

$\hat{\rho}_{\Psi}(t)=\iint P(t, \Psi, \bar{\Psi}) \frac{|\Psi\rangle\langle\bar{\Psi}|}{\langle\bar{\Psi} \mid \Psi\rangle} d[\Psi] d[\bar{\Psi}]$

The resulting Fokker-Planck equation for the positive distribution $P(t, \Psi, \bar{\Psi})$ has only second order derivative terms. Sometimes the notation $\Psi^{+}=\bar{\Psi}^{*}$ is used to indicate the stochastic field that corresponds to the hermitian conjugate of $\Psi$.

The equation for the Wigner function $W(t, \Psi)$ also contains third and fourth order derivative terms, which may be neglected at large photon number. The resultant Fokker-Planck equation in either case, can be converted into equivalent Ito stochastic equations for the phase space variables $\Psi$ (and $\bar{\Psi}$ ). Physical quantities can be calculated by forming the average over many stochastic realizations, or paths, in phase-space. For example, in the positive- $P$ representation, $\left\langle\bar{\Psi}^{*} \Psi\right\rangle_{\text {stochastic }}=\left\langle\widehat{\Psi}^{\dagger} \widehat{\Psi}\right\rangle_{\text {quantum }}$, while in the Wigner representation, $\left\langle\Psi^{*} \Psi\right\rangle_{\text {stochastic }}=\frac{1}{2}\left\langle\widehat{\Psi}^{\dagger} \widehat{\Psi}+\widehat{\Psi} \widehat{\Psi}^{\dagger}\right\rangle_{\text {quantum }}$.

It should be clear from this that the positive- $P$ representation directly generates an intensity corresponding to the usual normally ordered intensity that is detected in direct 
photodetection. The Wigner representation, however, generates an intensity result that includes some vacuum fluctuations. In a computer simulation with a finite number $M$ of modes, we must correct the Wigner result by subtracting $M / 2$ from any simulated photon number, or $v M / 2$ from any calculated photon flux, in order to obtain the direct photodetection result. For the calculation of a homodyne measurement, the Wigner method will give the most directly suitable result with symmetric ordering. In this case it is the positive- $P$ representation that will need correction terms added to it. Once these corrections are made, either method will give similar results, although the sampling error may not be identical.

\section{A. Modified nonlinear Schrödinger equation}

Standard custom in fiber optics applications 31 involves using the propagative reference frame with the normalized variables: $\tau=(t-x / v) / t_{0}$ and $\zeta=x / x_{0}$, where $t_{0}$ is a typical pulse duration used for scaling purposes, and $x_{0}=t_{0}^{2} /\left|k^{\prime \prime}\right| \sim 1 \mathrm{~km}$ for dispersion shifted fiber. This change of variables is useful only when slowly varying second order derivatives involving $\zeta$ can be neglected, which occurs for $v t_{0} / x_{0} \ll 1$. For typical values of the parameters used in experiments, this inequality is often well-satisfied $\left(v t_{0} \sim 10^{-4} \mathrm{~m}\right)$. To make it simpler to compare with this usage, we will make the same transformation for the stochastic equations that are equivalent to our complete operator equations, and scale the variables used in a dimensionless form.

For definiteness, we will now focus on the spatially uniform case. The resultant equation, which includes gain and loss, is a Raman-modified nonlinear Schrödinger (NLS) equation with stochastic noise terms:

$$
\begin{aligned}
\frac{\partial}{\partial \zeta} \phi(\tau, \zeta) & =-\int_{-\infty}^{\infty} d \tau^{\prime} g\left(\tau-\tau^{\prime}\right) \phi\left(\tau^{\prime}, \zeta\right)+\Gamma(\tau, \zeta) \\
& +\left[ \pm \frac{i}{2} \frac{\partial^{2} \phi}{\partial \tau^{2}}+i \int_{-\infty}^{\infty} d \tau^{\prime} h\left(\tau-\tau^{\prime}\right) \phi^{*}\left(\tau^{\prime}, \zeta\right) \phi\left(\tau^{\prime}, \zeta\right)+\Gamma^{R}(\tau, \zeta)\right] \phi(\tau, \zeta),
\end{aligned}
$$

where $\phi=\Psi \sqrt{v t_{0} / \bar{n}}$ is a dimensionless photon field amplitude. The photon flux is $|\phi|^{2} \bar{n} / t_{0}$, and $\bar{n}=\left|k^{\prime \prime}\right| A c /\left(n_{2} \hbar \omega_{c}^{2} t_{0}\right)=v^{2} t_{0} / \chi x_{0}$ is the typical number of photons in a soliton pulse of width $t_{0}$, for scaling purposes. The positive sign in front of the second derivative term applies for anomalous dispersion $\left(k^{\prime \prime}<0\right)$, which occurs for longer wavelengths, and the negative sign applies for normal dispersion $\left(k^{\prime \prime}>0\right)$. A similar equation is obtained in the positive- $P$ case, except that $\phi^{*}$ and $\Gamma^{R *}(\tau, \zeta)$ are replaced by non-complex-conjugate fields denoted $\phi^{+}$and $\Gamma^{R+}(\tau, \zeta)$ respectively:

$$
\begin{aligned}
\frac{\partial}{\partial \zeta} \phi^{+}(\tau, \zeta) & =-\int_{-\infty}^{\infty} d \tau^{\prime} g^{*}\left(\tau-\tau^{\prime}\right) \phi^{+}\left(\tau^{\prime}, \zeta\right)+\Gamma^{+}(\tau, \zeta) \\
& +\left[\mp \frac{i}{2} \frac{\partial^{2} \phi^{+}}{\partial \tau^{2}}-i \int_{-\infty}^{\infty} d \tau^{\prime} h^{*}\left(\tau-\tau^{\prime}\right) \phi\left(\tau^{\prime}, \zeta\right) \phi^{+}\left(\tau^{\prime}, \zeta\right)+\Gamma^{+R}(\tau, \zeta)\right] \phi^{+}(\tau, \zeta)
\end{aligned}
$$

The equations in $\phi$ and $\phi^{+}$both have the same additive noises and identical mean values, only differing by the independent parts of the multiplicative noise sources - which therefore generate nonclassical quantum statistics. 
The causal linear response function $g(\tau)$ is defined as:

$g(\tau)=\frac{\gamma\left(\tau t_{0}\right) x_{0}}{v}$.

If the Fourier transform of this function is $\widetilde{g}(\Omega)=g(\Omega)+i g^{\prime}(\Omega)$, then we can relate this to dimensionless intensity gain $\alpha^{G}(\Omega)$ and loss $\alpha^{A}(\Omega)$, at a relative (dimensionless) detuning of $\Omega$, by:

$2 g(\Omega)=\alpha^{A}(\Omega)-\alpha^{G}(\Omega)$.

The causal nonlinear response function $h(\tau)$ is normalized so that $\int h(\tau) d \tau=1$, and it includes both electronic and Raman nonlinearities:

$h(\tau)=h^{E}(\tau)+h^{R}(\tau)=\frac{\bar{n} x_{0} \chi\left(\tau t_{0}\right)}{v^{2}}$.

The Raman response function $h^{R}(\tau)$ causes effects like the soliton self-frequency shift倩. The response function Fourier transform is given by:

$\widetilde{h}(\Omega)=\int d t \exp (i \Omega \tau) h(\tau)=h^{\prime}(\Omega)+i h^{\prime \prime}(\Omega)$.

This definition has the property that the value of $\widetilde{h}(\Omega)=\widetilde{h}\left(\omega t_{0}\right)$ is a dimensionless number, which depends on the frequency $\omega$ only, independent of the time-scale used for normalization. The Raman gain, whose spectrum has been extensively measured 22, can be modeled as a sum of $n$ Lorentzians, as explained in section 3 and as illustrated in Fig. 1.

This expansion as $n$ Lorentzians gives a response function of the form

$h^{R}\left(t / t_{0}\right)=\Theta(t) \sum_{j=0}^{n} F_{j} \delta_{j} t_{0} \exp \left(-\delta_{j} t\right) \sin \left(\omega_{j} t\right)$,

It is most convenient to express these in terms of dimensionless parameters $\Omega_{j}=\omega_{j} t_{0}$ and $\Delta_{j}=\delta_{j} t_{0}$, giving:

$h^{R}(\tau)=\Theta(\tau) \sum_{j=0}^{n} F_{j} \Delta_{j} \exp \left(-\Delta_{j} \tau\right) \sin \left(\Omega_{j} \tau\right)$.

Here $\Delta_{j}$ are the equivalent dimensionless widths (corresponding to damping), and the $\Omega_{j}$ are the dimensionless center frequencies, all in normalized units. It is useful to compare these results with the dimensionless Raman gain $\alpha^{R}(\Omega)$, normalized following Gordon 32 , which uses a characteristic time-scale of $t_{0}$. The relationship of macroscopic coupling $R(\omega)$ to measured Raman gain $\alpha^{R}(\Omega)$ is $R^{2}(\omega)=\chi \alpha^{R}\left(\omega t_{0}\right) / 2 \pi$. It follows that the dimensionless gain function is

$\alpha^{R}(\Omega)=2\left|h^{\prime \prime}(\Omega)\right|$.

These stochastic partial differential equations can be discretized and, without any further approximation, can be numerically simulated 3335 using a split-step Fourier integration routine. The equations include all the currently known noise physics significant in soliton propagation, including effects like the soliton self-frequency shift. Guided acoustic wave Brillouin scattering2 202 noise sources are included in the Raman gain function. These have little effect on the position of an isolated soliton, but are important for long-range soliton collision effect.26 that occur in pulse-trains. 


\section{B. Initial conditions}

The initial conditions for the calculations could involve any required quantum state, if the $+P$ representation is used. In the case of the Wigner equations, only a subset of possible states can be represented with a positive probability distribution. The usual initial condition is the multi-mode coherent state, since this is the simplest model for the output of modelocked lasers. In general, there could be extra technical noise. We note that the choice of a coherent state is the simplest known model of a laser sources. To represent this in the positive- $P$ distribution is simple; one just takes

$\phi_{P}(\tau, 0)=\left[\phi_{P}^{+}(\tau, 0)\right]^{*}=\langle\widehat{\phi}(\tau, 0)\rangle$.

In the Wigner case, which corresponds to symmetric operator ordering, one must also include complex quantum vacuum fluctuations, in order to correctly represent operator fields. For coherent inputs, the Wigner vacuum fluctuations are Gaussian, and are correlated as

$$
\begin{aligned}
\left\langle\phi_{W}(\tau, 0)\right\rangle & =\langle\widehat{\phi}(\tau, 0)\rangle \\
\left\langle\Delta \phi_{W}(\tau, 0) \Delta \phi_{W}^{*}\left(\tau^{\prime}, 0\right)\right\rangle & =\frac{1}{2 \bar{n}} \delta\left(\tau-\tau^{\prime}\right) .
\end{aligned}
$$

We note that these equations imply that an appropriate correction is made for losses at the input interface, so that the mean-field evolution is known at the fiber input face.

\section{Wigner noise}

Both fiber loss and the presence of a gain medium contribute quantum noise to the equations in this symmetrically ordered representation. The complex gain/absorption noise enters the Wigner equation through an additive stochastic term $\Gamma$, whose correlations are obtained by averaging the normally and anti-normally ordered reservoir correlation functions given previously, together with appropriate variable changes. This symmetrically ordered noise source is present for both gain and loss reservoirs. Thus,

$$
\left\langle\Gamma(\Omega, \zeta) \Gamma^{*}\left(\Omega^{\prime}, \zeta^{\prime}\right)\right\rangle=\frac{\left(\alpha^{G}(\Omega)+\alpha^{A}(\Omega)\right)}{2 \bar{n}} \delta\left(\zeta-\zeta^{\prime}\right) \delta\left(\Omega-\Omega^{\prime}\right),
$$

where $\Gamma(\Omega, \zeta)$ is the Fourier transform of the noise source:

$$
\begin{aligned}
\Gamma(\Omega, \zeta) & =\frac{1}{\sqrt{2 \pi}} \int_{-\infty}^{\infty} d \tau \Gamma(\tau, \zeta) \exp (i \Omega \tau) \\
\Gamma^{*}(\Omega, \zeta) & =\frac{1}{\sqrt{2 \pi}} \int_{-\infty}^{\infty} d \tau \Gamma^{*}(\tau, \zeta) \exp (-i \Omega \tau)
\end{aligned}
$$

Similarly, the real Raman noise, which appears as a multiplicative stochastic variable $\Gamma^{R}$, has correlations

$$
\left\langle\Gamma^{R}(\Omega, \zeta) \Gamma^{R *}\left(\Omega^{\prime}, \zeta^{\prime}\right)\right\rangle=\frac{\alpha^{R}(|\Omega|)}{\bar{n}}\left[n_{t h}\left(|\Omega| / t_{0}\right)+\frac{1}{2}\right] \delta\left(\zeta-\zeta^{\prime}\right) \delta\left(\Omega-\Omega^{\prime}\right) .
$$


Thus the Raman noise is strongly temperature-dependent, but it also contains a spontaneous component which provides vacuum fluctuations even at $T=0$. Since the spontaneous component can occur through coupling to either a gain or a loss reservoir, in a symmetrically ordered representation, it is present for both positive and negative frequency detunings.

It must be remembered here that the noise terms in the Wigner representation do not correspond to normally ordered correlations, and so have no direct interpretation in terms of photodetection experiments. Any predictions made with this method of calculation need to be corrected by subtracting the appropriate commutators, to convert the results into a normally ordered form. This is the reason why there is no obvious distinction between the amplifier and absorber cases.

\section{D. $+P$ noise}

The positive P-representation is a useful alternative strategy, because it does not require truncation of higher order derivatives in a Fokker-Planck equation, and corresponds directly to observable normally ordered, time-ordered operator correlations. It has no vacuum fluctuation terms. Provided the phase-space boundary terms are negligible, one can then obtain a set of c-number stochastic differential equations in a phase-space of double the usual classical dimensions. These are very similar to the classical equations. Here the additive stochastic term is as before, except it only depends on the gain term $\alpha^{G}$; the conjugate term $\Gamma^{*}$ is used in the $\phi^{+}$equation:

$$
\left\langle\Gamma(\Omega, \zeta) \Gamma^{*}\left(\Omega^{\prime}, \zeta^{\prime}\right)\right\rangle=\frac{\alpha^{G}(\Omega)}{\bar{n}} \delta\left(\zeta-\zeta^{\prime}\right) \delta\left(\Omega-\Omega^{\prime}\right) .
$$

Since this representation is normally ordered, the only noise sources present are due to the gain reservoirs. There is no vacuum noise term for the absorbing reservoirs, because absorption simply maps a coherent state into another coherent state.

The complex terms $\Gamma^{R}, \Gamma^{R+}$ include both Raman and electronic terms (through $h^{\prime}(\Omega)$ ). As elsewhere in this paper, we regard $\Gamma^{R+}(\Omega, \zeta)$ as a hermitian conjugate Fourier transform (with the opposite sign frequency exponent):

$\Gamma^{+}(\Omega, \zeta)=\frac{1}{\sqrt{2 \pi}} \int_{-\infty}^{\infty} d \tau \Gamma^{+}(\tau, \zeta) \exp (-i \Omega \tau)$.

This quantity is not the same as $\Gamma^{R *}(\Omega, \zeta)$, since it involves a noise source that is in general independent. In some cases, where classical noise is dominant (and nonclassical squeezing is negligible), we can ignore this fact, and approximately set $\Gamma^{R+}(\Omega, \zeta)=\Gamma^{R *}(\Omega, \zeta)$. More generally, we obtain the following results:

$$
\begin{aligned}
\left\langle\Gamma^{R}(\Omega, \zeta) \Gamma^{R}\left(\Omega^{\prime}, \zeta^{\prime}\right)\right\rangle & =\delta\left(\zeta-\zeta^{\prime}\right) \delta\left(\Omega+\Omega^{\prime}\right)\left\{\left[n_{t h}\left(|\Omega| / t_{0}\right)+1 / 2\right] \alpha^{R}(|\Omega|)-i h^{\prime}(\Omega)\right\} / \bar{n} \\
\left\langle\Gamma^{R+}\left(\Omega^{\prime}, \zeta^{\prime}\right) \Gamma^{R}(\Omega, \zeta)\right\rangle & =\delta\left(\zeta-\zeta^{\prime}\right) \delta\left(\Omega-\Omega^{\prime}\right)\left[n_{t h}\left(|\Omega| / t_{0}\right)+\Theta(-\Omega)\right] \alpha^{R}(|\Omega|) / \bar{n} .
\end{aligned}
$$

This equation is an expected result, since it states that when $\Omega<0$ the spectral intensity of noise due to the Stokes process, in which a photon is down-shifted in frequency by an amount $\Omega$ with the production of a phonon of the same frequency, is proportional to $n_{t h}+1$. 
However the anti-Stokes process in which a phonon is absorbed $(\Omega>0)$ is only proportional to $n_{t h}$. Thus, at low temperatures the only direct noise effect is that due to the Stokes process, which can be interpreted physically as originating in spontaneous photon emission, detectable through photodetection.

As one might expect, the two forms of equation are identical at high phonon occupation numbers, when classical noise is so large that it obscures the differences due to the operator orderings of the two representations. Another, less obvious, result is that the two equations have identical additive noise sources, provided the gain and loss are balanced. To understand this, we can see that in the absence of any net gain or loss, the differences in the operator correlations due to ordering is a constant, contained in the initial conditions.

However, when gain and loss are not equal, the additive noise sources are quite different. In particular, the Wigner representation has noise contributions from both types of reservoir. On the other hand, the normally ordered $+P$ method only leads to additive noise when there is a real fluorescent field present, which is detectable through photodetection. This corresponds physically to some kind of gain, either due to the presence of an amplifier, or through Raman effects.

In general, the Wigner and $+P$ reservoir correlations are obtainable simply by examining the expectation values of the Heisenberg reservoir terms, with symmetric and normal ordering respectively. The additional term proportional to $h^{\prime}(\Omega)$ in the $+P$ noise terms is due to dispersive nonlinear effects, and gives rise to a nonclassical noise source which is responsible for the observed quantum squeezing effects. Extensions required to treat polarization dependent Raman scattering are given elsewhere 14 .

\section{Conclusions}

Our major conclusion is that quantum noise effects due to the intrinsic finite-temperature phonon reservoirs and finite bandwidth amplification or absorption can be readily modeled using stochastic equations. The equations themselves have the usual classical form, together with correction terms that we can describe as quantum noise terms. The precise form of the correction terms depends in detail on the representation employed (although this difference is purely due to operator ordering), as well as the physical origin of the reservoir couplings. These correction terms can be non-Markovian or nonuniform in space. The generation of the corresponding stochastic noises is a straightforward numerical procedure, and generally much simpler than the use of noncommuting operators. By contrast, the original operator equations have no practical numerical solution in most cases, due to the exponential growth of the dimension of the underlying Hilbert space with the number of modes and photons involved.

Detailed applications to short-pulse soliton communications will be given in a subsequent paper6. In general, the increasing bandwidth, reducing pulse energies and greater demands placed on fiber communications and sensors mean that these quantum limits are becoming increasingly important. Already, limits set by quantum amplifiers are known to have great significance in long-distance laser-amplified communications systems. We note that the quantum theory given here also establishes the levels of quantum noise in silica fibers in more general situations. Examples of this are for dispersion-managed fiber communications 36,37 , and for fiber ring lasers with relatively low gain 38 . Similarly, these equations set the limits 
for experiments using spectral filtering and related techniques to generate sub-shot-noise pulses 39,0 in optical fibers.

\section{ACKNOWLEDGMENTS}

We would like to acknowledge helpful comments on this paper by Wai S. Man. 


\section{REFERENCES}

1. S. J. Carter, P. D. Drummond, M. D. Reid, and R. M. Shelby, "Squeezing of Quantum Solitons," Physical Review Letters 58, 1841-1844 (1987).

2. P. D. Drummond and S. J. Carter, "Quantum-Field Theory of Squeezing in Solitons," Journal of the Optical Society of America B 4, 1565-1573 (1987).

3. M. Rosenbluh and R. M. Shelby, "Squeezed Optical Solitons", Phys. Rev. Lett. 66, 153156 (1991); P.D. Drummond, R. M. Shelby, S. R. Friberg and Y. Yamamoto, "Quantum solitons in optical fibres," Nature 365, 307-313 (1993).

4. J. P. Gordon and H. A. Haus, "Random Walk of Coherently Amplified Solitons in Optical Fiber Transmission," Optics Letters 11, 665-667 (1986).

5. H. A. Haus and W. S. Wong, "Solitons in Optical Communications," Reviews of Modern Physics 68, 423-444 (1996).

6. P. D. Drummond and J. F. Corney, "Quantum noise in optical fibers II: Raman jitter in soliton communications," submitted to Journal of the Optical Society of America B.

7. P. D. Drummond, "Electromagnetic quantization in dispersive inhomogeneous nonlinear dielectrics," Physical Review A 42, 6845-6857 (1990).

8. P. D. Drummond and S. J. Carter, "Quantum-Field Theory of Squeezing in Solitons," Journal of the Optical Society of America B 4, 1565-1573 (1987); P. D. Drummond, S. J. Carter, and R. M. Shelby, "Time Dependence of Quantum Fluctuations in Solitons," Optics Letters 14, 373-375 (1989).

9. B. Yurke and M. J. Potasek, "Solution to the Initial Value Problem for the Quantum Nonlinear Schroedinger Equation," Journal of the Optical Society of America B 6, 1227-1238 (1989).

10. P. D. Drummond and M. Hillery, "Quantum theory of dispersive electromagnetic modes", Phys. Rev. A 59, 691-707 (1999).

11. E. Power, S. Zienau, "Coulomb gauge in nonrelativistic quantum electrodynamics and the shape of spectral lines", Philos. Trans. Roy. Soc. Lond. A251, 427 -454 (1959); R. Loudon, The Quantum Theory of Light (Clarendon Press, Oxford, 1983).

12. M. Hillery and L. D. Mlodinow, "Quantization of electrodynamics in nonlinear dielectric media", Phys. Rev. A30, 1860 (1984).

13. N. Bloembergen, Nonlinear Optics, (Benjamin, New York, 1965).

14. P. D. Drummond, "Quantum Theory of Fiber-Optics and Solitons", in Coherence and Quantum Optics VII, J. Eberly, L. Mandel and E. Wolf (Eds), 323-332 (Plenum Press, New York, 1996).

15. G. P. Agrawal, Nonlinear Fiber Optics, 2nd ed. (Academic Press, 1995), pp 28-59.

16. S. J. Carter and P. D. Drummond, "Squeezed Quantum Solitons and Raman Noise," Physical Review Letters 67, 3757-3760 (1991).

17. F. X. Kartner, D. J. Dougherty, H. A. Haus, and E. P. Ippen, "Raman Noise and Soliton Squeezing," Journal of the Optical Society of America B 11, 1267-1276 (1994).

18. Y. Lai and S.-S. Yu, "General Quantum Theory of Nonlinear Optical-Pulse Propagation," Physical Review A 51, 817-829 (1995); S.-S. Yu and Y. Lai, "Impacts of SelfRaman Effect and Third-Order Dispersion on Pulse Squeezed State Generation Using Optical Fibers," Journal of the Optical Society of America B 12, 2340-2346 (1995).

19. T. von Foerster and R. J. Glauber, "Quantum Theory of Light Propagation in Amplifying Media," Physical Review A 3, 1484-1511 (1971); I. A. Walmsley and M. G. Raymer, 
"Observation of Macroscopic Quantum Fluctuations in Stimulated Raman Scattering," Phys. Rev. Lett. 50, 962-965 (1983).

20. M. D. Levenson, Introduction to Nonlinear Laser Spectroscopy (Academic Press, New York, 1982).

21. P. Dean, "The Vibrational Properties of Disordered Systems: Numerical Studies", Rev. Mod. Phys. 44, 127 (1972).

22. R. H. Stolen, C. Lee, and R. K. Jain, "Development of the Stimulated Raman Spectrum in Single-Mode Silica Fibers," Journal of the Optical Society of America B 1, 652-657 (1984); D. J. Dougherty, F. X. Kartner, H. A. Haus, and E. P. Ippen, "Measurement of the Raman Gain Spectrum of Optical Fibers," Optics Letters 20, 31-33 (1995); R. H. Stolen, J. P. Gordon, W. J. Tomlinson, and H. A. Haus, "Raman Response Function of Silica-Core Fibers," Journal of the Optical Society of America B 6, 1159-1166 (1989).

23. R. M. Shelby, M. D. Levenson, and P. W. Bayer, "Guided acoustic-wave Brillouin scattering", Physical Review B 31, 5244-5252 (1985).

24. R. M. Shelby, P. D. Drummond, and S. J. Carter, "Phase-Noise Scaling in Quantum Soliton Propagation," Physical Review A 42, 2966-2796 (1990).

25. K. Bergman, H. A. Haus, and M. Shirasaki, "Analysis and Measurement of GAWBS Spectrum in a Nonlinear Fiber Ring," Applied Physics B 55, 242-249 (1992).

26. K. Smith and L. F. Mollenauer, "Experimental observation of soliton interaction over long fiber paths: discovery of a long-range interaction," Optics Letters 14, 1284-1286 (1989); E. M. Dianov, A. V. Luchnikov, A. N. Pilipetskii, and A. M. Prokhorov, "LongRange Interaction of Picosecond Solitons Through Excitation of Acoustic Waves in Optical Fibers," Applied Physics B 54, 175-180 (1992).

27. S. H. Perlmutter, M. D. Levenson, R. M. Shelby and M. B. Weissman, "Inverse-PowerLaw Light scattering in Fused-Silica Optical Fiber", Phys. Rev. Lett. 61, 1388-1391 (1988); "Polarization properties of quasielastic light scattering in fused-silica optical fiber", Physical Review B 42, 5294-5305 (1990).

28. R. J. Mears, L. Reekie, I. M. Jauncey, D. N. Payne, "Low-noise erbium-doped fibre

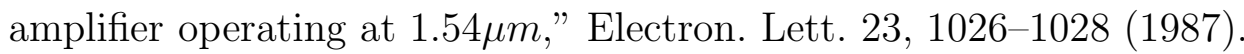

29. E. Desurvire, Erbium-Doped Fiber Amplifiers, Principles and Applications (Wiley, New York, 1993).

30. P. D. Drummond and M. G. Raymer, "Quantum theory of propagation of nonclassical radiation in a near-resonant medium" Physical Review A 44, 2072-2085 (1991).

31. L. F. Mollenauer, "Solitons in optical fibers and the soliton laser", Philosophical Transactions of the Royal Society of London A 315, 435 (1985); L. F. Mollenauer, R. H. Stolen, and J. P. Gordon, "Experimental observation of picosecond pulse narrowing and solitons in optical fibers," Physical Review Letters 45, 1095-1098 (1980).

32. J. P. Gordon, "Theory of the Soliton Self-Frequency Shift", Optics Letters 11, 662-664 (1986); F. M. Mitschjke and L. F. Mollenauer, "Discovery of the Soliton Self-Frequency Shift", Optics Lett. 11, 659-661 (1986).

33. P. D. Drummond and A. D. Hardman, "Simulation of Quantum Effects in RamanActive Waveguides," Europhysics Letters 21, 279-284 (1993); P. D. Drummond and W. Man, "Quantum Noise in Reversible Soliton Logic," Optics Communications 105, 99-103 (1994).

34. S. J. Carter, "Quantum Theory of Nonlinear Fiber Optics: Phase-Space representa- 
tions," Physical Review A 51, 3274-3301 (1995).

35. M. J. Werner and P. D. Drummond, "Robust algorithms for solving stochastic partial differential equations", J. Comp. Phys. 132, 312-326 (1997).

36. N. J. Smith, N. J. Doran, W. Forysiak, and F. M. Knox, "Soliton transmission using periodic dispersion compensation", IEEE J. Lightwave Technol., 15, 1808-1822 (1997).

37. T. I. Lakoba, and D. J. Kaup, "Influence of the Raman effect on dispersion-managed solitons and their interchannel collisions", Optics Letts. 24, 808-810 (1999).

38. S. Namiki, C. X. Yu, and H. A. Haus, "Observation of nearly quantum-limited timing jitter in an all-fiber ring laser", Journal of the Optical Society of America B 13, 28172823 (1996); B. C. Collings, K. Bergman, and W. H. Knox, "Stable multigigahertz pulsetrain formation in a short-cavity passively harmonic mode-locked erbium/ytterbium fiber laser", Opt. Letts. 23, 123-125 (1998).

39. S. R. Friberg, S. Machida, M. J. Werner, A. Levanon and T. Mukai, "Observation of Optical Soliton Photon-Number Squeezing," Phys. Rev. Lett. 77, 3775-3778 (1996); S. Spalter, M. Burk, U. Strossner, M. Bohm, A. Sizmann, and G. Leuchs, "Photon number squeezing of spectrally filtered sub-picosecond optical solitons," Europhys. Lett. 38, 335-340 (1997); D. Krylov and K. Bergman, "Amplitude-squeezed solitons from an asymmetric fiber interferometer", Opt. Letts. 23, 1390-1392, (1998).

40. M. J. Werner, "Raman-induced photon correlations in optical fiber solitons", Phys. Rev. A 60, R781-R784 (1999). 


\section{FIGURES}

Fig. 1. The parallel polarization Raman gain $\left|\Im\left\{\tilde{h}\left(\omega t_{0}\right)\right\}\right|=\left|h^{\prime \prime}\left(\omega t_{0}\right)\right|$ for the 11-Lorentzian model (continuous lines) and the single-Lorentzian model (dashed lines), for a temperature of $T=300 \mathrm{~K}$. 


\section{TABLES}

Table 1. Fitting parameters for the 11-Lorentzian model of the Raman gain function $h^{R}\left(t / t_{0}\right)$. All frequencies are in T.rad/s.

\begin{tabular}{r|r|r|r|}
\hline \hline$j$ & $F_{j}$ & $\omega_{j}$ & $\delta_{j}$ \\
\hline 0 & 0.16 & 0.005 & 0.005 \\
\hline 1 & -0.3545 & 0.3341 & 8.0078 \\
\hline 2 & 1.2874 & 26.1129 & 46.6540 \\
\hline 3 & -1.4763 & 32.7138 & 33.0592 \\
\hline 4 & 1.0422 & 40.4917 & 30.2293 \\
\hline 5 & -0.4520 & 45.4704 & 23.6997 \\
\hline 6 & 0.1623 & 93.0111 & 2.1382 \\
\hline 7 & 1.3446 & 99.1746 & 26.7883 \\
\hline 8 & -0.8401 & 100.274 & 13.8984 \\
\hline 9 & -0.5613 & 114.6250 & 33.9373 \\
\hline 10 & 0.0906 & 151.4672 & 8.3649 \\
\hline \hline
\end{tabular}




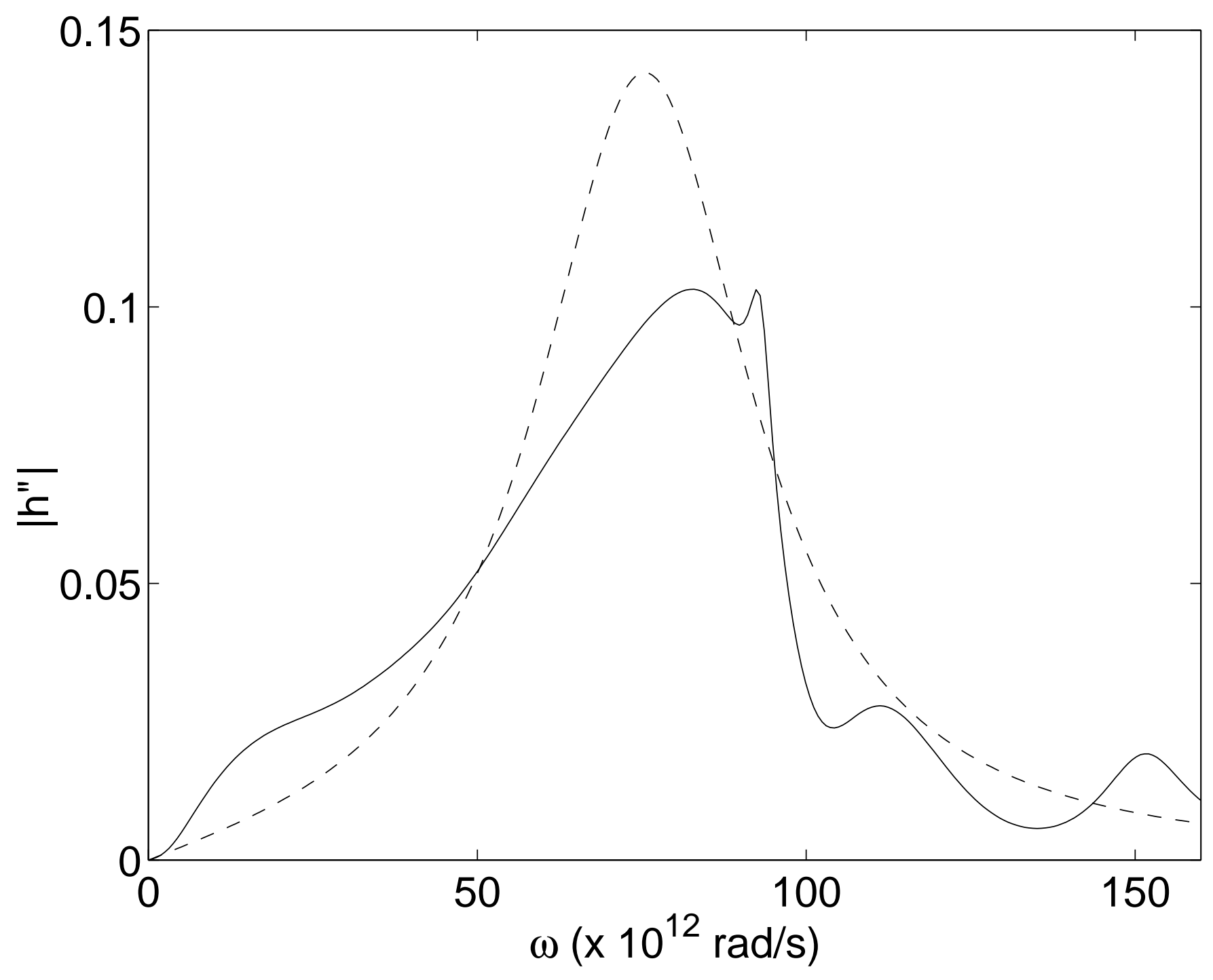

